\title{
Phase diagrams of cholesteric liquid crystals obtained with a generalized Landau-de Gennes theory
}

\author{
by L. LONGA \\ Jagellonian University, Department of Statistical Physics, Reymonta 4, \\ Kraków, Poland \\ D. MONSELESAN and H.-R. TREBIN \\ Institut für Theoretische und Angewandte Physik der Universität Stuttgart, \\ Pfaffenwaldring 57, D-7000 Stuttgart 80, F.R. Germany \\ Phase diagrams of chiral nematic liquid crystals are studied within the frame- \\ work of a generalized Landau-Ginzburg-de Gennes theory. Using the parametriz- \\ ation of Grebel, Hornreich, and Shtrikman for the tensor order parameter $\mathbf{Q}$, all \\ relevant elastic terms are included for the helicoidal phase and the blue phases of \\ chiral nematic liquid crystals up to fourth order in $\mathbf{Q}$ and its gradient $\partial \mathbf{Q}$. The \\ influence of the additional elastic terms on the phase diagrams of the chiral \\ nematic phases is then investigated. The theory correctly describes the variation of \\ the pitch with temperature and the induced biaxiality of the cholesteric phase. The \\ results resolve the discrepancies encountered by Hornreich and Shtrikman in the \\ comparison of experiment and theory. New features in the topology of the phase \\ diagrams of blue phases, like re-entrant phase transitions, are predicted.
}

\section{Introduction}

Though the cholesteric and blue phases of chiral nematic liquid crystals have been intensively studied for more than a century $[1,2]$, only recently has it become evident that three different blue phases can be distinguished: blue phase I (BPI) with a body-centred structure, blue phase II (BP II) with a simple cubic structure and the still mysterious amorphous blue phase III (BP III). The analysis of BPI and BP II by Landau-Ginzburg-de Gennes theory [3-6] has been very successful in explaining the basic features of the experimental observations. In particular, it has been shown that the properties of BPI and BP II are consistent with a space group assignment of body-centred cubic $\mathrm{O}^{8}\left(\mathrm{I}_{4}, 32\right)$ for BP I and simple cubic $\mathrm{O}^{2}\left(\mathrm{P}_{2}{ }_{2} 32\right)$ for BP II. Up to three different modifications of the $\mathrm{O}^{8}$ phase have been predicted theoretically. The free energy difference between the different structures has been predicted to be extremely small, which indicates that higher order terms in the free energy density expansion [7] could play an important role, leading to qualitative changes in the topology of cholesteric phase diagrams.

It is the purpose of this paper to study this suggestion theoretically.

\section{Landau-Ginzburg-de Gennes theory}

The fundamental ingredient of the Landau-Ginzburg-de Gennes theory is a second rank, symmetric and traceless tensor field, $\mathbf{Q}(\mathbf{r})$, with cartesian components $Q_{\alpha \beta}(\mathbf{r})(\alpha, \beta=x, y, z)$. The tensor vanishes in the isotropic phase and thus serves as an order parameter. Macroscopically, it may be associated with the anisotropic part 
of the dielectric and diamagnetic susceptibilities. A spatial dependence of $\mathbf{Q}$ requires elastic terms, i.e. those proportional to $\partial \mathbf{Q}$ (in components $\partial_{\alpha} Q_{\beta \gamma}$ ) in a LandauGinzburg expansion of the free energy. To the lowest orders in $\partial \mathbf{Q}$ and in the absence of electric and magnetic fields, the free energy is

$$
\begin{aligned}
F & =F^{2}+F^{3}+F^{4}, \\
F^{2} & =\frac{1}{v} \int_{v} d v \frac{1}{2}\left[a Q_{i j}^{2}+c_{1}\left(\partial_{l} Q_{i j}\right)^{2}+c_{2} \partial_{i} Q_{i j} \partial_{l} Q_{l j}-2 d e_{i j l} Q_{i n} \partial_{j} Q_{l n}\right], \\
F^{3} & =-\beta \frac{1}{v} \int_{v} d v Q_{i j} Q_{j l} Q_{l i}, \\
F^{4} & =\gamma \frac{1}{v} \int_{v} d v\left(Q_{i j}^{2}\right)^{2} .
\end{aligned}
$$

Here $a$ is proportional to a reduced temperature, $c_{1}, c_{2}, d, \beta$ and $\gamma$ are temperature independent parameters, and $e_{i j l}$ is the Levi-Civita tensor. The Einstein summation convention over repeated indices is applied. The term proportional to $d$ violates parity and is responsible for the formation of a helical ground state.

The stability of expansion (1) requires that

$$
\gamma \geqslant 0, \quad c_{1} \geqslant 0, \quad c_{1}+\frac{2}{3} c_{2} \geqslant 0 .
$$

In order to study the minima of $F$ for periodic structures of the classical helicoidal cholesteric (C) phase, of BP I and BP II, it is convenient to expand $\mathbf{Q}(\mathbf{r})$ in a Fourier series,

$$
\mathbf{Q}(\mathbf{r})=\sum_{* \mathbf{k}} \frac{1}{N_{* \mathbf{k}}^{1 / 2}}\left(\sum_{\mathbf{k} \in *_{\mathbf{k}}} \mathbf{Q}(\mathbf{k}) \exp (i \mathbf{k} \cdot \mathbf{r})\right)
$$

where

$$
\mathbf{Q}(\mathbf{k})=\left\{\sum_{m=-2} Q_{m, \mathbf{k}}^{[2] *}(\mathbf{k}) \mathbf{e}_{m, \mathbf{k}}^{[2]}\right\} .
$$

Here $\mathbf{k}$ is a reciprocal lattice vector, ${ }^{*} \mathbf{k}=\left\{\mathbf{k}^{\prime} \mid \mathbf{k}^{\prime}=S \mathbf{k},\{S \mid \mathbf{t}\} \in G\right\}$ is the star of $\mathbf{k}$, $G$ denotes a cubic space group, $N_{* \mathbf{k}}$ is the number of prongs of the $\operatorname{star}{ }^{*} \mathbf{k}, Q_{m, \mathbf{k}}^{[2]}(\mathbf{k})$ are coefficients in the expansion and, finally,

$$
\begin{aligned}
\mathbf{e}_{0, \mathbf{k}}^{[2]} & =\frac{1}{6^{1 / 2}}\left(\begin{array}{rrr}
-1 & 0 & 0 \\
0 & -1 & 0 \\
0 & 0 & 2
\end{array}\right), \\
\mathbf{e}_{ \pm 1, \mathbf{k}}^{[2]} & =\frac{1}{2}\left(\begin{array}{rrr}
0 & 0 & \pm i \\
0 & 0 & 1 \\
\pm i & 1 & 0
\end{array}\right), \\
\mathbf{e}_{ \pm 2, \mathbf{k}}^{[2]]} & =\frac{1}{2}\left(\begin{array}{rrr}
1 & \mp i & 0 \\
\mp i & -1 & 0 \\
0 & 0 & 0
\end{array}\right)
\end{aligned}
$$


are second rank tensors represented in an orthogonal, right handed coordinate system with the quantization axis parallel to the unit vector $\hat{\mathbf{k}}$.

Writing

$$
Q_{m, S \mathbf{k}}^{[2]}(S \mathbf{k})=\left|Q_{m, S \mathbf{k}}^{[2]}(S \mathbf{k})\right| \exp \left(i \psi_{m, S \mathbf{k}}\right)
$$

the space group symmetry requires that amplitudes and phases of modes $\mathbf{k}$ and $S \mathbf{k}$ belonging to the same star *k fulfil the conditions

$$
\left|Q_{m, \mathbf{k}}^{[2]}(\mathbf{k})\right|=\left|Q_{m, S \mathbf{k}}^{[2]}(S \mathbf{k})\right|=Q_{m}(\sigma) \geqslant 0
$$

and

$$
\psi_{m, \mathbf{k}}-\psi_{m, S \mathbf{k}}=\mathbf{k} \cdot \mathbf{t}-m \phi_{S^{-1}, \mathbf{k}}
$$

where $\sigma=\left(h^{2}+k^{2}+l^{2}\right)^{1 / 2}$ is the star index and $Q_{S^{-1, \mathbf{k}}}$ is a phase, which is determined by the space group symmetry of the order parameter field $(3 a)$.

In order to make calculations feasible we must decide how many different symmetry allowed stars we have to retain in the expansion (3). We must also determine the relative phases $\mathbf{k} \cdot \mathbf{t}-m \phi_{S-1, \hat{\mathbf{k}}}$. The information about the relevant $\mathbf{k}$ vectors may be partially deduced from experiment $[1,2]$. In particular, for the usual cholesteric (C) phase the tensor order parameter takes the simple form of a transverse spiral along the $\hat{z}$ axis

$$
\mathbf{Q}_{\mathrm{C}}(\mathbf{r})=-Q_{0}(0) \mathbf{e}_{0, \mathbf{z}}^{[2]}+\frac{1}{2^{1 / 2}} Q_{2}(1)\left[\exp i\left(k_{\mathrm{C}} z+\phi\right) \mathbf{e}_{2, \mathbf{z}}^{[2]}+\text { c.c. }\right]
$$

where c.c. denotes the complex conjugate, $\phi$ is an arbitrary phase expressing a freedom of choice of the reference frame and $k_{\mathrm{C}}=2 \pi / p$ for the wavevector associated with the cholesteric pitch $p$. Thus, only two amplitudes $Q_{0}(0)$ and $Q_{2}(1)$ must be determined in order to find the explicit form of $\mathbf{Q}_{C}(\mathbf{r})$. The twist induced simple spiral of the $C$ phase causes the order parameter $\mathbf{Q}$ to be biaxial. A measure of the biaxiality, the so-called asymmetry parameter $\eta=\eta(\mathbf{r})(0 \leqslant \eta \leqslant 1)$ may be defined by diagonalizing the order parameter (5) [8]

$$
\left[\mathbf{Q}_{\mathrm{C}}\right]_{\text {diag }}=\frac{1}{6^{1 / 2}\left[Q_{0}(0)+3^{1 / 2} Q_{2}(1)\right]}\left(\begin{array}{ccc}
\frac{1}{2}(-1-\eta) & 0 & 0 \\
0 & \frac{1}{2}(-1+\eta) & 0 \\
0 & 0 & 1
\end{array}\right)
$$

where

$$
\eta=1-\frac{4 Q_{0}(0)}{Q_{0}(0)+3^{1 / 2} Q_{2}(1)}
$$

In considering the blue phases, we shall concentrate on $\mathrm{O}^{2}, \mathrm{O}^{5}$ and $\mathrm{O}^{8}$ cubic symmetries. Following the notation of Hornreich and Shtrikman we first introduce the reduced parameters

$$
\begin{array}{rlrl}
Q_{m}(\sigma) & =\frac{\beta}{\gamma 6^{1 / 2}} \mu_{m}(\sigma), & F_{\text {red }} & =\frac{36 \gamma^{3}}{\beta^{4}} F, \\
q_{\mathrm{C}} & =\frac{d}{c_{1}}, & \frac{1}{4} t & =\frac{3 \gamma}{\beta^{2}} a, \\
\mathbf{q} & =\frac{\mathbf{k}}{q_{c}}=q[h, k, l], & \frac{1}{4} \xi_{\mathrm{R}}^{2} & =\frac{3 \gamma}{\beta^{2}} c_{1}, \\
\kappa & =q_{\mathrm{C}} \xi_{R} . &
\end{array}
$$




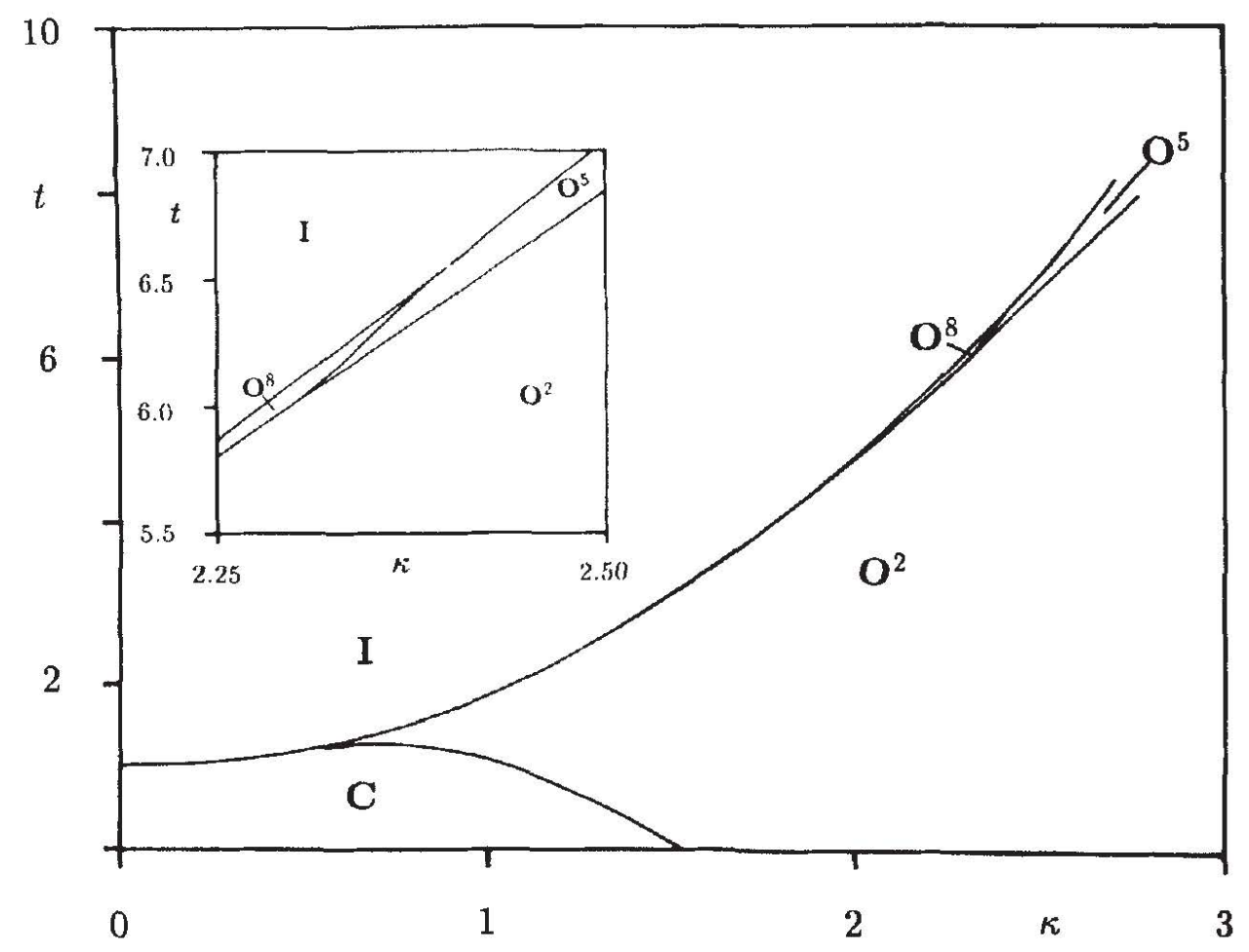

Figure 1. Phase diagrams predicted by Grebel et al. [5], shown with all relevant phases included.

These definitions are introduced to eliminate the freedom of scale for $\boldsymbol{F}$ and $\mathbf{Q}$ in equation (1). The meaning of the parameters in equation (7) is explained in [5]. In particular, $\kappa$ is the chirality parameter and $\xi_{\mathrm{R}}$ is the correlation length at the isotropicracemic $\left(q_{\mathrm{C}}=\kappa=0\right)$ phase boundary.

All of the relevant features of BPI and BP II phase diagrams can now be reproduced by taking into account in the expansion $(3 a)$ only the first two stars for the $\mathrm{O}^{2}, \mathrm{O}^{5}$ and $\mathrm{O}^{8}$ space groups, i.e. $[1,0,0],[1,1,0]$ for $\mathrm{O}^{2},[1,1,0]$ for $\mathrm{O}^{5}$ and $[1,1,0],[2,0,0]$ for $\mathrm{O}^{8}$. With these assumptions and definitions the free energy in equation (1) is expressed in terms of $\mu_{m}(\sigma)(m=0, \pm 1, \pm 2 ; \sigma=1,2,4), \kappa, q$ and $t$, where $\mu_{m}(\sigma)$ and $q$ are variational parameters. Retaining the dominant $m=2$ order parameter and minimizing $F_{\text {red }}$ with respect to $q$ and $\mu_{2}(\sigma)$ finally yields the phase diagrams presented in figure 1 [5].

\section{Generalized Landau-Ginzburg-de Gennes theory}

We now study how elastic terms of higher order than in expansion (1) effect the topology of the phase diagram.

The dominant higher order invariants are cubic terms of the form $\mathbf{Q} \partial \mathbf{Q} \partial \mathbf{Q}$, where $\mathbf{Q} \partial \mathbf{Q} \partial \mathbf{Q}$ denotes the class of all independent $\mathbf{S O}(3)$ invariants built up from the tensors $Q_{\alpha \beta} \partial_{\rho} Q_{\gamma \delta} \partial_{\sigma} Q_{\mu \nu}$. For the stabilization of the free energy expansion we must also retain $\mathbf{Q Q} \partial \mathbf{Q} \partial \mathbf{Q}$ terms. Without proof we mention that the class of pseudoscalar invariants $\mathbf{Q} \mathbf{Q} \partial \mathbf{Q}, \mathbf{Q Q Q} \partial \mathbf{Q}$ and $\partial \mathbf{Q} \partial \mathbf{Q} \partial \mathbf{Q}$ may safely be disregarded in the limit of weak chiral interactions, which are discussed here. Also the terms $\partial \mathbf{Q} \partial \mathbf{Q} \partial \mathbf{Q} \partial \mathbf{Q}$ are not expected to be relevant for the type of long wavelength structural organization observed in C, BP I and BP II. On the other hand, the phase transitions discussed here are first order, which means that the non-chiral elastic terms proportional to $\mathbf{Q}$ and QQ may be important. 
These have been enumerated in our recent paper [7]. Here we showed that the free energy expansion up to order $\mathbf{Q} \mathbf{Q} \partial \mathbf{Q} \partial \mathbf{Q}$ contains, in addition to $F^{2}, F^{3}$ and $F^{4}$ terms (see Equation (1)) 19 more elastic terms, denoted in [7] as $\left[L_{\alpha}^{(3)}\right], \alpha=1, \ldots, 6$ and $\left[L_{\beta}^{(4)}\right], \beta=1, \ldots, 13$. Though three surface relations eliminate one invariant $\left[L_{\alpha}^{(3)}\right]$ and two invariants $\left[L_{\beta}^{(4)}\right]$, studies of the free energy expansion with 16 free parameters appear hopeless. It is important, therefore, to identify among $\left[L_{\alpha}^{(i)}\right], i=3,4$ the relevant terms. Unique features of these terms come to light, if we realize that the dominant local distortion in $\mathrm{C}, \mathrm{O}^{2}, \mathrm{O}^{5}$ and $\mathrm{O}^{8}$ structures is the twist. Thus, we neglect those elastic terms, which in the mean field decomposition into splay, bend and twist (see table 4 of [7]) do not contain the twist component. The validity of this assumption is supported by explicit calculations, given in the Appendix, of the individual contributions $\left[L_{\alpha}^{(i)}\right], i=3,4$, to the free energies of $\mathrm{C}, \mathrm{O}^{2}, \mathrm{O}^{5}$ and $\mathrm{O}^{8}$ phases. Using the results given there we identify as dominant the elastic terms

$$
\begin{gathered}
L_{4}^{(3)} Q_{i j} \partial_{k} Q_{i l} \partial_{k} Q_{j l}, \\
L_{2}^{(4)} Q_{i j} Q_{i j} \partial_{k} Q_{l m} \partial_{k} Q_{l m}, \\
L_{7}^{(4)} Q_{i k} Q_{j k} \partial_{l} Q_{i m} \partial_{l} Q_{j m} .
\end{gathered}
$$

The stability of the corresponding extended Landau-Ginzburg-de Gennes free energy requires, in addition to conditions (2), that

$$
L_{2}^{(4)} \geqslant 0, \quad\left(L_{2}^{(4)}+L_{7}^{(4)}\right)-\frac{\left(L_{4}^{(3)}\right)^{2}}{2 c_{1}} \geqslant 0 .
$$

Using the convenient notation

$$
\lambda=\frac{d^{2}}{c_{1}^{2} \beta} L_{4}^{(3)}, \quad u=\frac{d^{2}}{c_{1}^{2} \gamma}\left(L_{2}^{(4)}+\frac{1}{2} L_{7}^{(4)}\right), \quad v=\frac{d^{2}}{c_{1}^{2} \gamma} L_{7}^{(4)},
$$

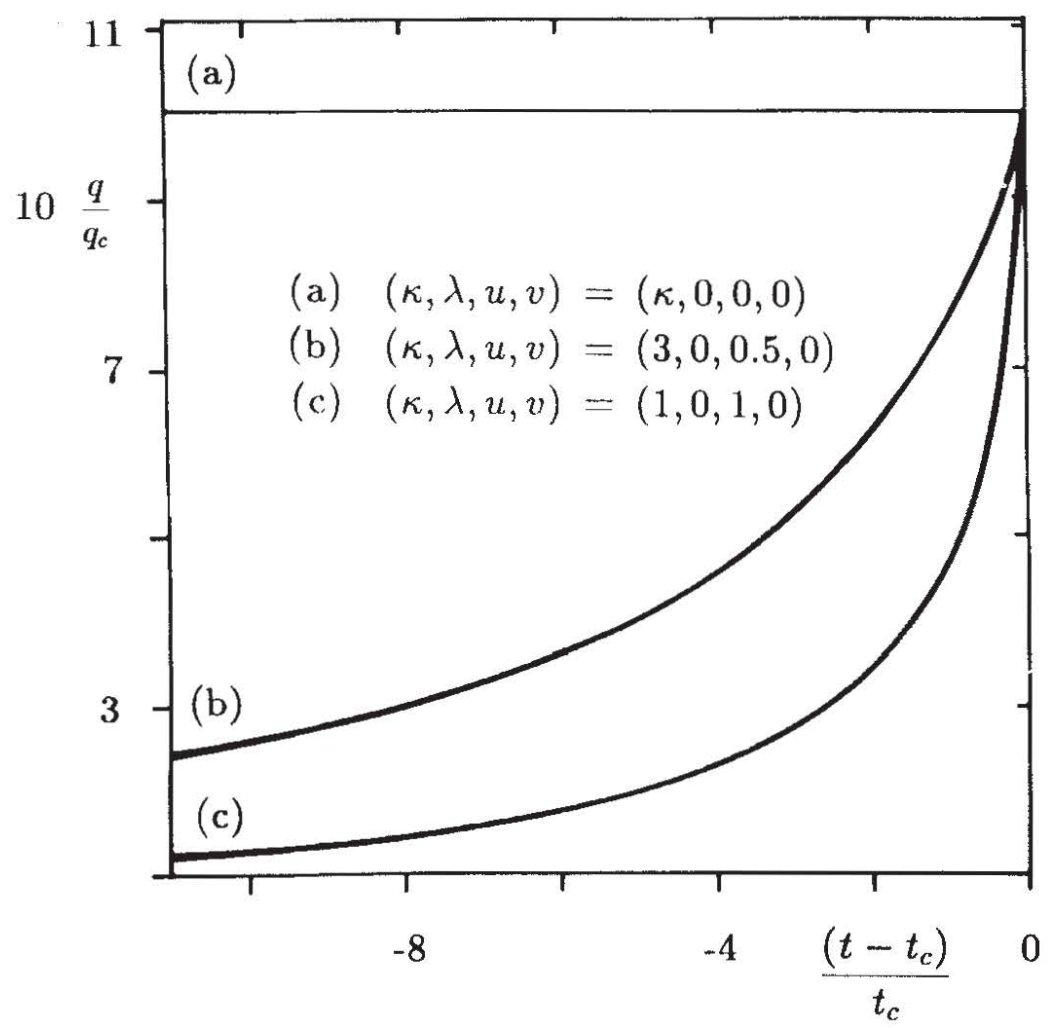

Figure 2. Chirality $q / q_{\mathrm{C}}$ versus temperature; $t_{\mathrm{C}}$ denotes the cholesteric-isotropic transition temperature. 


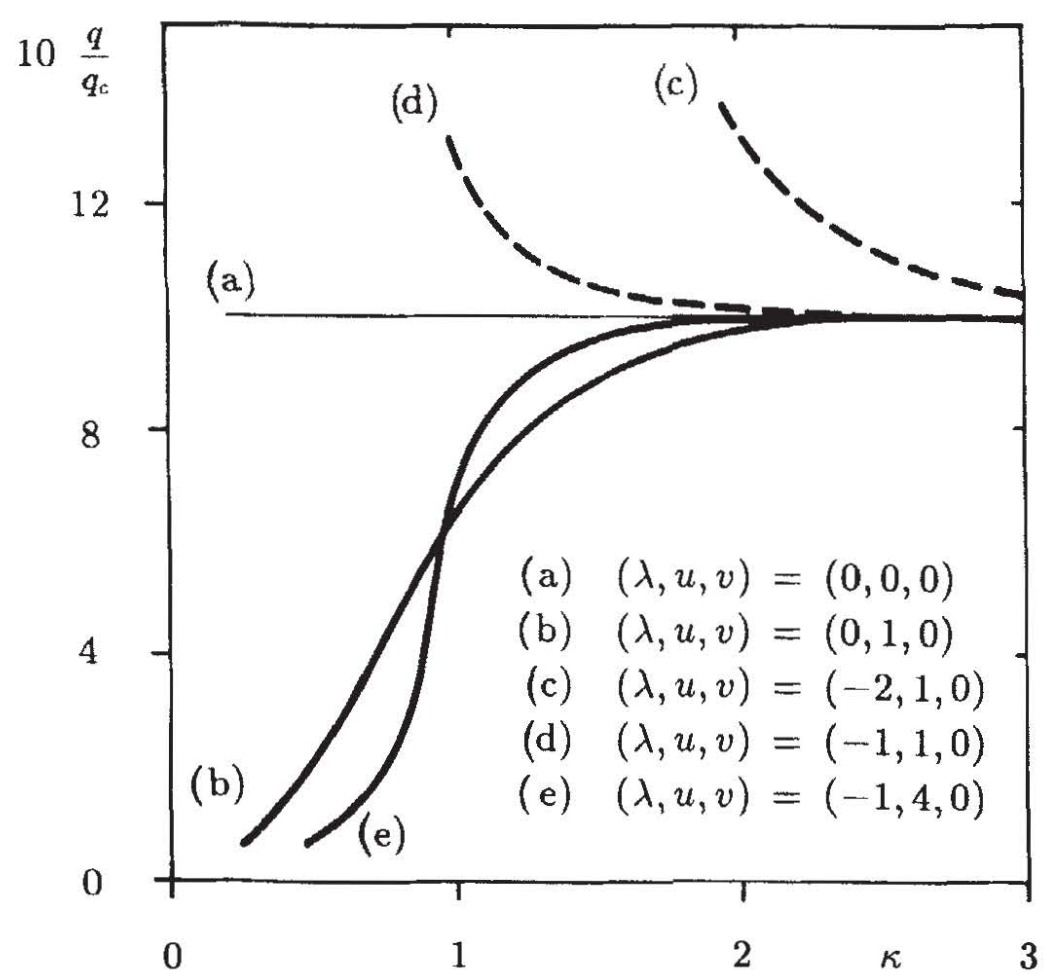

Figure 3. Chirality $q / q_{\mathrm{C}}$ versus chirality parameter $\kappa$ at the cholesteric-isotropic transition.

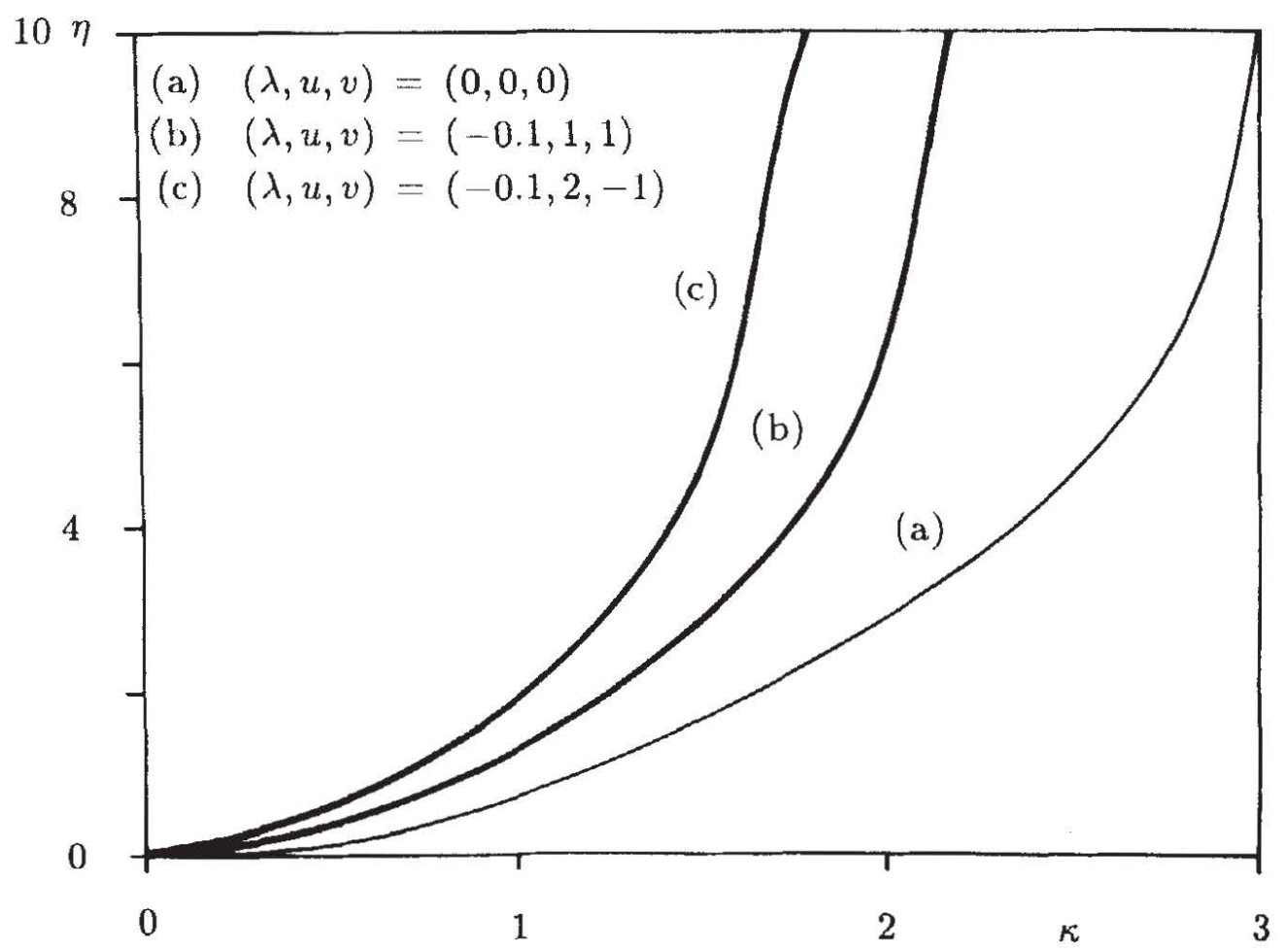

Figure 4. Biaxiality versus chirality parameter $\kappa$ at the cholesteric-isotropic transition.

we can now minimize the free energies, listed in the Appendix, with respect to $q$ and $\mu_{2}(\sigma)$.

The results, calculated at the low temperature phase transition to the $\mathrm{C}$ phase, are shown in figures $2-4$, with chirality $q / q_{\mathrm{C}}$ defined in close relation to experiment. As 


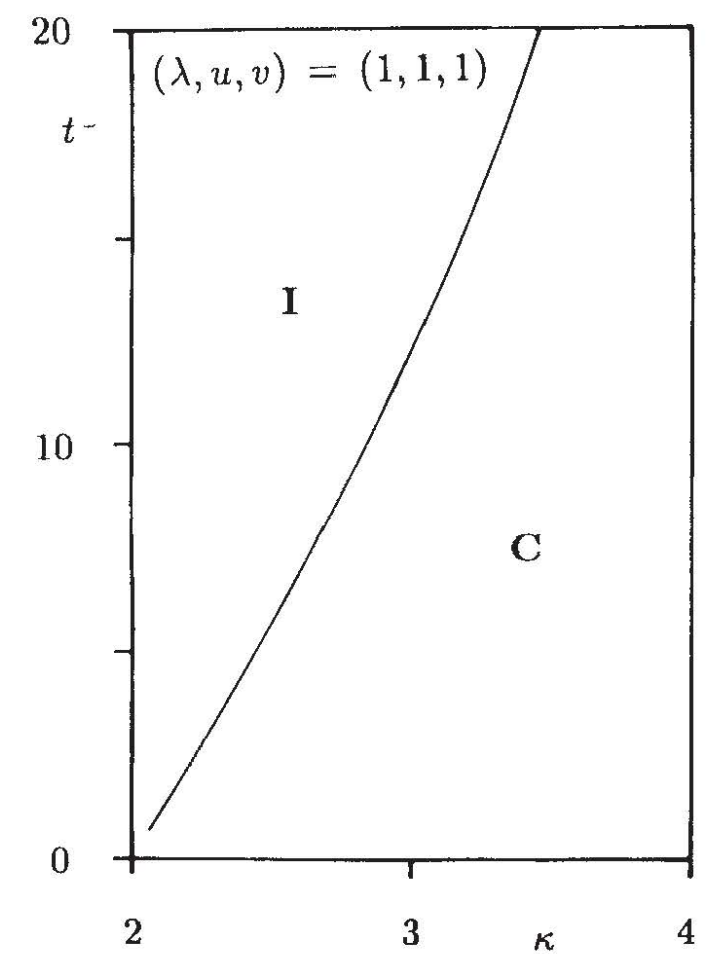

(a)

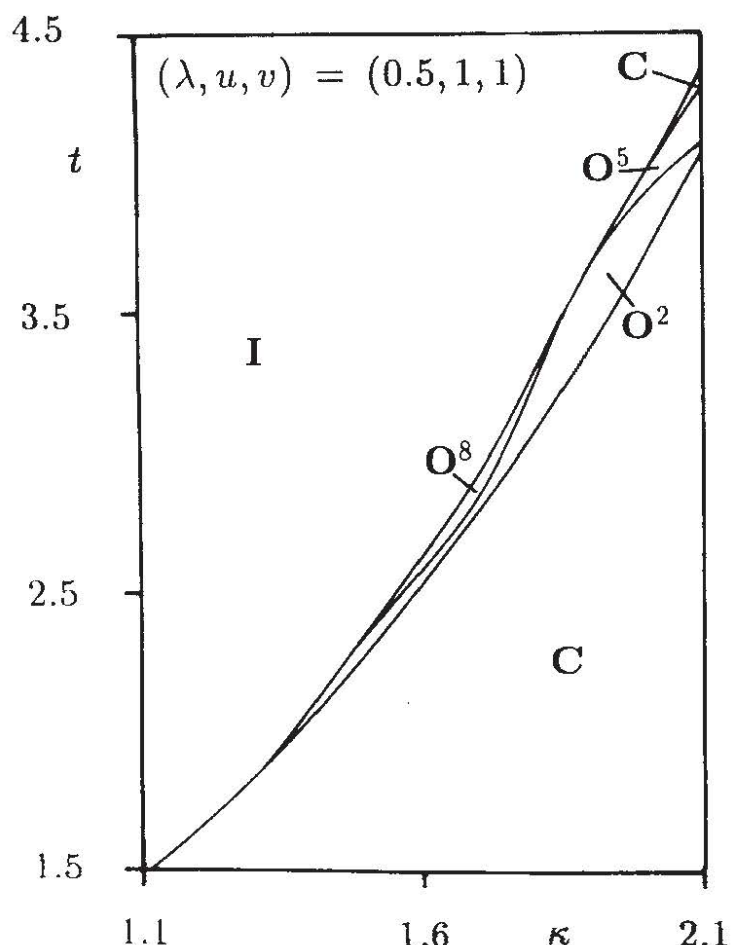

(c)

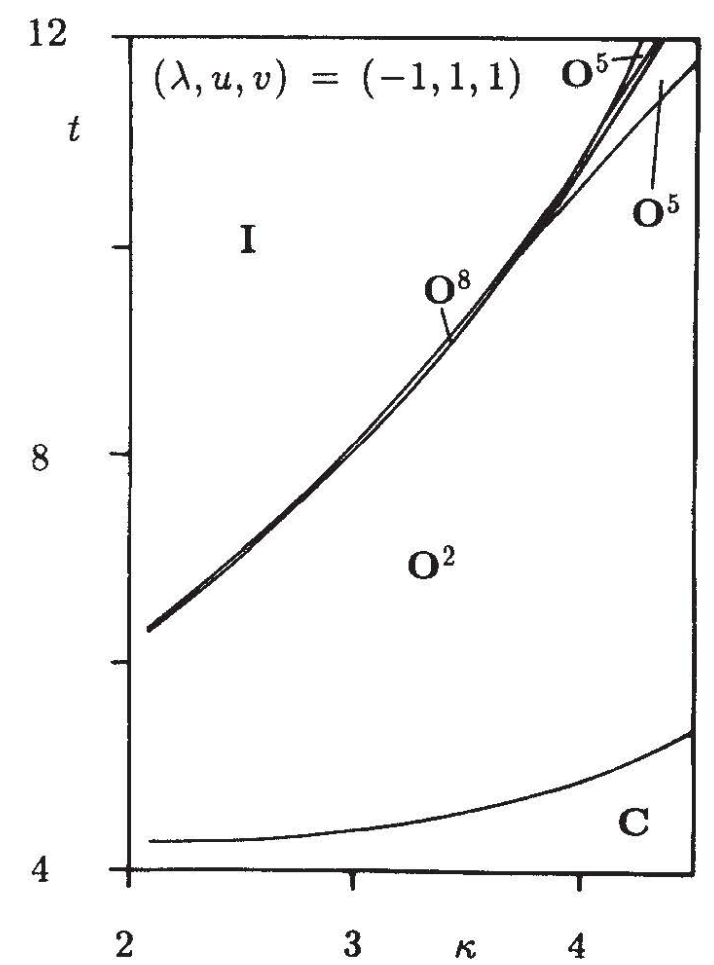

(b)

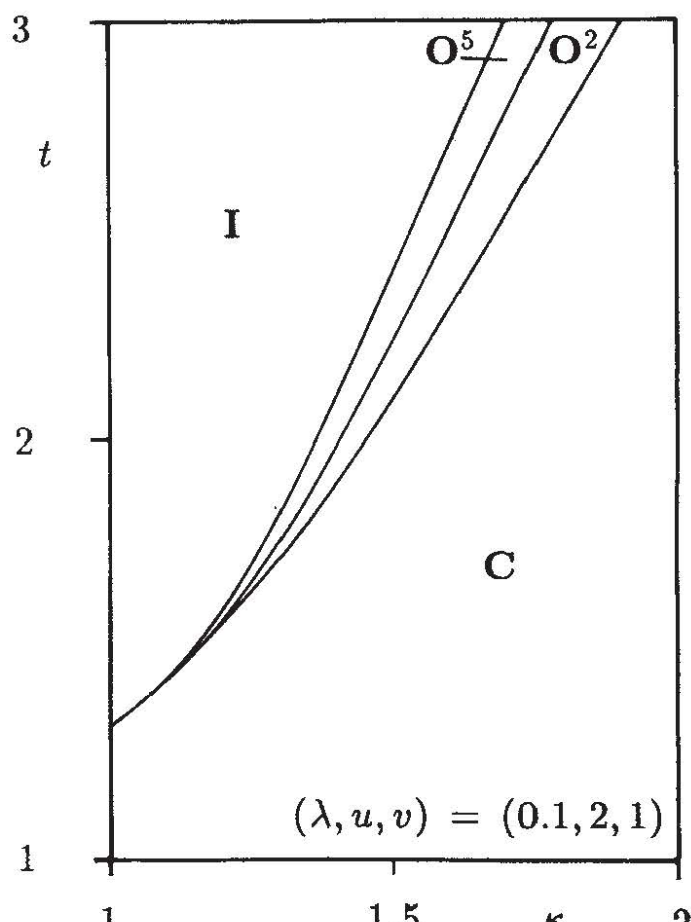

$(d)$

Figure 5. Possible modifications of phase diagrams given in figure 1, caused by the presence of higher order elastic terms.

seen from the figures, qualitatively new results are obtained for both cholesteric and blue phases. In particular, we find that the theory yields a strong dependence of pitch and asymmetry parameter of the $C$ phase on temperature and chirality (see figures 2-4). The trends are consistent with experimental observations $[8,9]$ and in contrast with the predictions of Hornreich-Shtrikman theory [10] (see branch $(a)$ in 
figures 2-4). Only the asymmetry parameter $\eta$, calculated at the isotropic-cholesteric transition, follows the trends found in [10]. When normalized to its value at the cholesteric-isotropic transition, it appears to be a universal function of the reduced temperature for fixed $\kappa$. The curves are essentially indistinguishable from those of Hornreich and Shtrikman (see figure 2, [10]).

Another interesting aspect is the behaviour of blue phase diagrams, figures $5(a)$ $(d)$. The inclusion of higher order terms may dramatically alter the phase sequence of blue phases and may even lead to re-entrant phases. The reason is the strong nonlinear coupling between $q$ and $\mu_{2}(\sigma)$. In this respect our re-entrant phases are very similar to those observed by Feldman et al. [11] under external, chiral strain. The important difference, however, is that in our model the chiral strain (i.e. the changes of $q$ ) is a thermodynamic variable, calculated at equilibrium.

This work is partially supported by the Alexander von Humboldt Foundation, by the Polish Project C.P.B.P.01.03, and by Deutsche Forschungsgemeinschaft.

\section{Appendix}

The free energies of $\mathrm{C}, \mathrm{O}^{2}, \mathrm{O}^{5}$ and $\mathrm{O}^{8}$ phases calculated for all invariants $\left[L_{\alpha}^{(\beta)}\right]$ [7]. For completeness the standard Landau-Ginzburg-de Gennes part (1) is also included (see [5]). The terms

$$
\begin{array}{ll}
\tilde{L}_{m}^{(3)}=\frac{q_{\mathrm{C}}^{2}}{\beta} L_{m}^{(3)}, & m=1, \ldots, 7, \\
\tilde{L}_{n}^{(4)}=\frac{q_{\mathrm{C}}^{2}}{\gamma} L_{n}^{(4)}, & n=1, \ldots, 15,
\end{array}
$$

denote the higher order material parameters in the rescaled free energies. These energies read:

$$
\begin{aligned}
F_{\mathrm{C}}= & \frac{1}{4} t \mu_{0}^{2}(0)+\frac{1}{4}\left[t-\kappa^{2}\right] \mu_{2}^{2}(1)+\mu_{0}^{3}(0)+\mu_{2}^{4}(1) \\
+ & \left(-3+\tilde{L}_{4}^{(3)} q^{2}+2 \tilde{L}_{7}^{(3)} q\right) \mu_{0}(0) \mu_{2}^{2}(1) \\
+ & {\left[2+\left(\tilde{L}_{2}^{(4)}+\frac{1}{6} \tilde{L}_{7}^{(4)}-\frac{1}{3} \tilde{L}_{11}^{(4)}\right) q^{2}+\frac{1}{6} \widetilde{L}_{14}^{(4)} q+\widetilde{L}_{15}^{(4)} q\right] \mu_{0}^{2}(0) \mu_{2}^{2}(1) } \\
& +\left[1+\left(\tilde{L}_{2}^{(4)}+\frac{1}{2} \tilde{L}_{7}^{(4)}\right) q^{2}-\frac{1}{2} \tilde{L}_{14}^{(4)} q+\tilde{L}_{15}^{(4)} q\right] \mu_{2}^{4}(1) \\
F_{\mathrm{O}^{5}}= & \frac{1}{4}\left[t-\kappa^{2}+\kappa^{2}(q-1)^{2}\right] \mu_{2}^{2}(2) \\
& +\frac{23(2)^{1 / 2}}{32}\left[-1+\left(1 \cdot 0000 \tilde{L}_{4}^{(3)}-1 \cdot 0000 \tilde{L}_{5}^{(3)}+1 \cdot 0000 \tilde{L}_{6}^{(3)}\right) q^{2}\right. \\
& \left.-1 \cdot 4142 \tilde{L}_{7}^{(3)} q\right] \mu_{2}^{3}(2) \\
& +\frac{499}{384}\left[1+\left(1 \cdot 7335 \tilde{L}_{2}^{(4)}-0 \cdot 2665 \tilde{L}_{3}^{(4)}+0 \cdot 6944 \tilde{L}_{7}^{(4)}-0 \cdot 3056 \tilde{L}_{8}^{(4)}\right.\right. \\
& \left.+0 \cdot 0431 \tilde{L}_{9}^{(4)}-0 \cdot 0862 \tilde{L}_{11}^{(4)}+0 \cdot 1333 \tilde{L}_{12}^{(4)}+0 \cdot 1333 \tilde{L}_{13}^{(4)}\right) q^{2} \\
& \left.-0 \cdot 4605 \tilde{L}_{14}^{(4)} q+1 \cdot 4142 \tilde{L}_{15}^{(4)} q\right] \mu_{2}^{4}(2)
\end{aligned}
$$




$$
\begin{aligned}
& F_{0^{2}}=\frac{1}{4}\left[t-\kappa^{2}+\kappa^{2}\left(\frac{q}{2^{1 / 2}}-1\right)^{2}\right] \mu_{2}^{2}(1)+\frac{1}{4}\left(\left[t-\kappa^{2}+\kappa^{2}(q-1)^{2}\right] \mu_{2}^{2}(2)\right. \\
& +\frac{23(2)^{1 / 2}}{32}\left[1+\left(-1 \cdot 0000 \tilde{L}_{4}^{(3)}+1 \cdot 0000 \tilde{L}_{5}^{(3)}-1 \cdot 0000 \tilde{L}_{6}^{(3)}\right) q^{2}\right. \\
& \left.+1 \cdot 4142 \tilde{L}_{7}^{(3)} q\right] \mu_{2}^{3}(2) \\
& +\frac{3\left[4+3(2)^{1 / 2}\right]}{8}\left[-1+\left(0.6667 \tilde{L}_{4}^{(3)}-0.6095 \tilde{L}_{5}^{(3)}+0.6095 \tilde{L}_{6}^{(3)}\right) q^{2}\right. \\
& \left.-1 \cdot 1381 \tilde{L}_{7}^{(3)} q\right] \mu_{2}^{2}(1) \mu_{2}(2) \\
& +\frac{499}{384}\left[1+\left(1 \cdot 7335 \tilde{L}_{2}^{(4)}-0 \cdot 2665 \tilde{L}_{3}^{(4)}+0.6944 \tilde{L}_{7}^{(4)}-0.3056 \tilde{L}_{8}^{(4)}\right.\right. \\
& \left.+0.0431 \tilde{L}_{9}^{(4)}-0.0862 \tilde{L}_{11}^{(4)}+0.1333 \tilde{L}_{12}^{(4)}+0.1333 \tilde{L}_{13}^{(4)}\right) q^{2} \\
& \text { - } \left.0.4605 \tilde{L}_{14}^{(4)} q+1.4142 \tilde{L}_{15}^{(4)} q\right] \mu_{2}^{4}(2) \\
& +\frac{139-12(2)^{1 / 2}}{48}\left[1+\left(1.2372 \tilde{L}_{2}^{(4)}-0.2544 \tilde{L}_{3}^{(4)}+0.6231 \tilde{L}_{7}^{(4)}-0.0984 \tilde{L}_{8}^{(4)}\right.\right. \\
& \left.-0.0072 \tilde{L}_{9}^{(4)}-0.0137 \tilde{L}_{11}^{(4)}+0 \cdot 1272 \tilde{L}_{12}^{(4)}+0.1314 \tilde{L}_{13}^{(4)}\right) q^{2} \\
& \left.-0.5063 \tilde{L}_{14}^{(4)} q+1.2071 \tilde{L}_{15}^{(4)} q\right] \mu_{2}^{2}(1) \mu_{2}(2)^{2} \\
& +\frac{12}{12}\left[1+\left(0.9231 \tilde{L}_{2}^{(4)}+0.0769 \tilde{L}_{3}^{(4)}+0.3077 \tilde{L}_{7}^{(4)}-0.1923 \tilde{L}_{8}^{(4)}\right.\right. \\
& \left.+0.0385 \tilde{L}_{9}^{(4)}-0.0769 \tilde{L}_{11}^{(4)}+0.0385 \tilde{L}_{12}^{(4)}+0.0385 \tilde{L}_{13}^{(4)}\right) q^{2} \\
& \left.-0.2692 \tilde{L}_{14}^{(4)} l+1.0000 \tilde{L}_{15}^{(4)} q\right] \mu_{2}(1)^{4}, \\
& F_{\mathrm{O}^{8}}=\frac{1}{4}\left[t-\kappa^{2}+\kappa^{2}\left(\frac{2 q}{2^{1 / 2}}-1\right)^{2}\right] \mu_{2}^{2}(4) \\
& +\frac{1}{4}\left[t-\kappa^{2}+\kappa^{2}(q-1)^{2}\right] \mu_{2}^{2}(2) \\
& +\frac{5}{8}\left[-1+\left(1 \cdot 0000 \tilde{L}_{4}^{(3)}-1 \cdot 0000 \tilde{L}_{5}^{(3)}+1 \cdot 0000 \tilde{L}_{6}^{(3)}\right) q^{2}-1 \cdot 4142 \tilde{L}_{7}^{(3)} q\right] \mu_{2}^{3}(2) \\
& +\frac{3\left[3+2(2)^{1 / 2}\right]}{8}\left[-1+\left(1 \cdot 3333 \tilde{L}_{4}^{(3)}-1 \cdot 2190 \tilde{L}_{5}^{(3)}+1 \cdot 2190 \tilde{L}_{6}^{(3)}\right) q^{2}\right. \\
& \left.-1.6095 \tilde{L}_{7}^{(3)} q\right] \mu_{2}(4) \mu_{2}^{2}(2) \\
& +\frac{449}{384}\left[1+\left(1 \cdot 8508 \tilde{L}_{2}^{(4)}-0 \cdot 1492 \tilde{L}_{3}^{(4)}+0.6537 \tilde{L}_{7}^{(4)}-0.3463 \tilde{L}_{8}^{(4)}\right.\right. \\
& \left.+0.0680 \tilde{L}_{9}^{(4)}-0.1359 \tilde{L}_{11}^{(4)}+0.0746 \tilde{L}_{12}^{(4)}+0.0746 \tilde{L}_{13}^{(4)}\right) q^{2} \\
& \left.-0.4142 \tilde{L}_{14}^{(4)} q+1.4142 \tilde{L}_{15}^{(4)} q\right] \mu_{2}^{4}(2) \\
& +\frac{24+17(2)^{1 / 2}}{48}\left[1+\left(1.4411 \tilde{L}_{2}^{(4)}-0.9731 \tilde{L}_{3}^{(4)}+1.0221 \tilde{L}_{7}^{(4)}-0 \cdot 1850 \tilde{L}_{8}^{(4)}\right.\right. \\
& \left.-0.0754 \tilde{L}_{9}^{(4)}+0 \cdot 1079 \tilde{L}_{11}^{(4)}+0.4865 \tilde{L}_{12}^{(4)}+0.5294 \tilde{L}_{13}^{(4)}\right) q^{2}
\end{aligned}
$$




$$
\begin{aligned}
& \left.-0.6963 \tilde{L}_{14}^{(4)} q+1.5607 \tilde{L}_{15}^{(4)} q\right] \mu_{2}(4) \mu_{2}^{3}(2) \\
& +\frac{151+12(2)^{1 / 2}}{48}\left[1+\left(2 \cdot 5232 \tilde{L}_{2}^{(4)}-0.4033 \tilde{L}_{3}^{(4)}+1.0432 \tilde{L}_{7}^{(4)}-0.4037 \tilde{L}_{8}^{(4)}\right.\right. \\
& \left.+0.0505 \tilde{L}_{9}^{(4)}-0 \cdot 1419 \tilde{L}_{11}^{(4)}+0 \cdot 2016 \tilde{L}_{12}^{(4)}+0.2384 \tilde{L}_{13}^{(4)}\right) q^{2} \\
& \left.-0.5707 \tilde{L}_{14}^{(4)} q+1 \cdot 7071 \tilde{L}_{15}^{(4)} q\right] \mu_{2}(4)^{2} \mu_{2}^{2}(2) \\
& +\frac{13}{12}\left[1+\left(3 \cdot 6923 \tilde{L}_{2}^{(4)}+0.3077 \tilde{L}_{3}^{(4)}+1 \cdot 2308 \tilde{L}_{7}^{(4)}-0.7692 \tilde{L}_{8}^{(4)}\right.\right. \\
& \left.+0 \cdot 1538 \tilde{L}_{9}^{(4)}-0 \cdot 3077 \tilde{L}_{11}^{(4)}+0 \cdot 1538 \tilde{L}_{12}^{(4)}+0 \cdot 1538 \tilde{L}_{13}^{(4)}\right) q^{2} \\
& \left.-0.5384 \tilde{L}_{14}^{(4)} q+2 \cdot 0000 \tilde{L}_{15}^{(4)} q\right] \mu_{2}^{4}(4)
\end{aligned}
$$

\section{References}

[1] Belyakov, V. A., and Dmitrienko, V. E., 1985, Usp. fiz. Nauk, 146, 369 (1985, Soviet Phys. Usp., 27, 535).

[2] Stegemeyer, H., Blümel, Th., Hiltrop, K., Onusseit, H., and Porsch, F., 1986, Liq. Crystals, 1, 3 (erratum: 1986, Liq. Crystals, 1, 305).

[3] Hornreich, R. M., and Shtrikman, S., 1981, Phys. Rev. A, 24, 635.

[4] Kleinert, H., and MaKı, K., 1981, Fortschr. Phys., 29, 219.

[5] Grebel, H., Hornreich, R. M., and Shtrikman, S., 1983, Phys. Rev. A, 28, 1114.

[6] Gremel, H., Hornreich, R. M., and Shtrikman, S., 1984, Phys. Rev. A, 30, 3264.

[7] Longa, L., Monselesan, D., and Trebin, H.-R., 1987, Liq. Crystals, $2,769$.

[8] Yaniv, Z., Chidichimo, G., and Doane, J. W., 1983, Phys. Rev. A, 28, 3012.

[9] Yaniv, Z., Neubert, M. E., and Doane, J. W., 1983, Liquid Crystals and Ordered Fluids, Vol. 4, edited by A. G. Griffin and J. Johnson (Plenum Press); 1983, Phys. Rev. A, 28, 3012.

[10] Hornreich, R. M., and Shtrikman, S., 1984, Phys. Rev. A, 29, 3444.

[11] Feldman, A. I., Crooker, P. P., and Goh, L. M., 1987, Phys. Rev. A, 35, 842. 